\title{
The Effect of Tegafur-Uracil on Survival in T Categories as Defined in the Eighth Edition of the TNM Classification: An Exploratory Analysis of Postoperative Adjuvant Tegafur-Uracil on Survival in Patients with Adenocarcinoma of the Lung
}

\author{
Masahiro Tsuboi $^{\mathrm{a}}$ Chikuma Hamada ${ }^{\mathrm{b}}$ Harubumi Kato ${ }^{c}$ Mitsuo Ohta ${ }^{\mathrm{d}}$ \\ ${ }^{a}$ Department of Thoracic Surgery, National Cancer Center Hospital East, Kashiwa, ${ }^{b}$ Faculty of Engineering, \\ Tokyo University of Science, Tokyo, 'Department of Thoracic Surgery, Niizashiki Central General Hospital, \\ Saitama, and ${ }^{\mathrm{d}}$ The Japan Lung Cancer Research Group, Fukuoka, Japan
}

\section{Keywords}

Tegafur-uracil · Lung adenocarcinoma · TNM classification · Postoperative adjuvant chemotherapy

\begin{abstract}
Background: Tegafur-uracil (UFT) improves survival in patients with stage I adenocarcinoma of the lung. We evaluated the effect of UFT on survival in maximum primary tumor diameter $(\mathrm{T})$ categories as defined in the eighth edition of the TNM Classification (TNM8). Methods: Tumors were subgrouped on the basis of T category (TNM8) as follows: T1a, T $\leq 1 \mathrm{~cm} ; \mathrm{T} 1 \mathrm{~b}, 1<\mathrm{T} \leq 2 \mathrm{~cm} ; \mathrm{T} 1 \mathrm{c}, 2<\mathrm{T} \leq 3 \mathrm{~cm} ; \mathrm{T} 2 \mathrm{a}, 3<\mathrm{T} \leq 4 \mathrm{~cm}$; $\mathrm{T} 2 \mathrm{~b}, 4<\mathrm{T} \leq 5 \mathrm{~cm} ; \mathrm{T} 3,5<\mathrm{T} \leq 7 \mathrm{~cm}$. Hazard ratios (HR) and $95 \%$ confidence intervals $(\mathrm{Cl})$ were estimated by Cox proportional hazard models. Results: UFT was associated with improved survival. The adjusted HRs were as follows: for T1a, 0.79 (95\% Cl 0.14-4.50); for T1b, 1.16 (95\% Cl 0.63-2.12); for T1c, 0.74 (95\% Cl 0.43-1.27); for T2a, 0.45 (95\% Cl 0.21-0.96); for T2b, 0.55 (95\% Cl 0.10-3.07), and for T3, 0.70 (95\% Cl 0.20-
\end{abstract}

2.50). Conclusions: The adjuvant chemotherapy with UFT tended to improve survival in patients with adenocarcinoma of the lung of each T category based on TNM8, except T1 b.

() 2017 The Author(s)

Published by S. Karger AG, Basel

\section{Introduction}

Non-small-cell lung cancer (NSCLC) accounts for about $85 \%$ of all lung cancer cases and its morbidity and mortality are very high globally, including in Japan [1, 2]. Patients with resectable NSCLC are treated by surgery and the 5-year survival rate after resection is about $70 \%$ overall, and 86.8 and $73.9 \%$ in those with early stage lung cancer (pathological stage IA and IB, respectively) [3].

In Japan, oral tegafur-uracil (UFT) has been investigated as a postoperative adjuvant chemotherapy for early NSCLC. The Japan Lung Cancer Research Group (JLCRG) performed a large phase III clinical trial com-

Masahiro Tsuboi

Department of Thoracic Surgery, National Cancer Center Hospital East 6-5-1, Kashiwanoha

Kashiwa, Chiba 277-8577 (Japan)

E-Mail mtsuboi@za2.so-net.ne.jp 
Fig. 1. Overall survival HRs based on each T category defined by the TNM8.

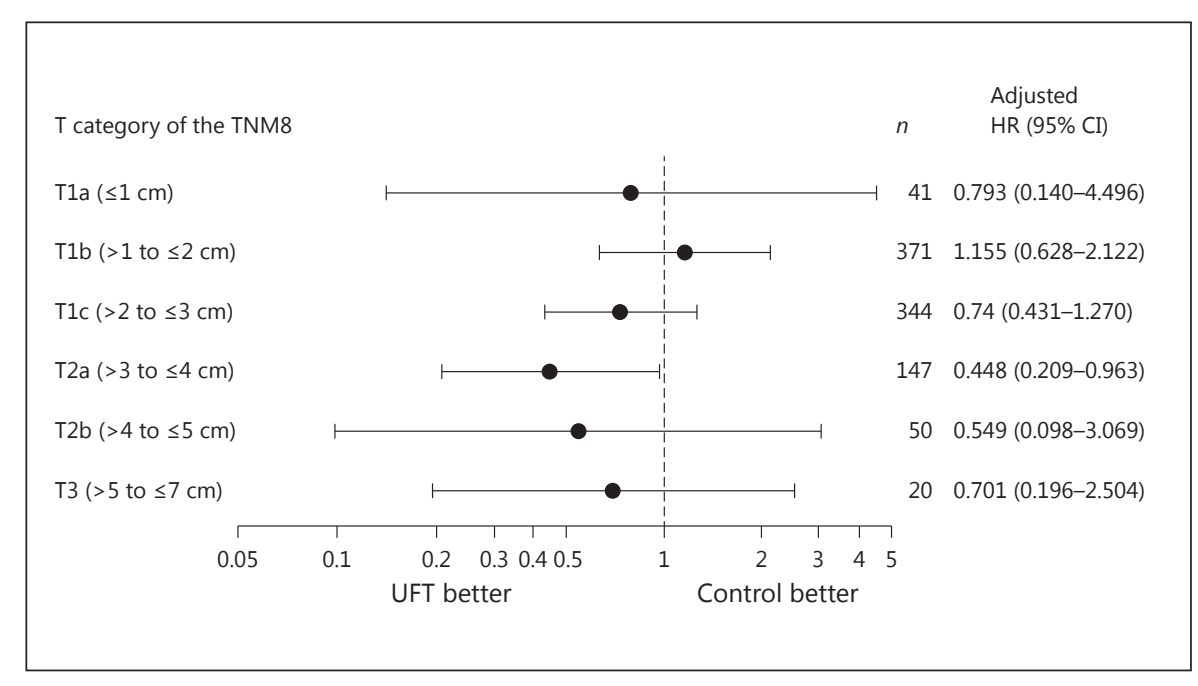

paring 2-year oral administration of UFT $\left(250 \mathrm{mg} / \mathrm{m}^{2} /\right.$ day) with surgery alone in patients with completely resected stage I adenocarcinoma of the lung. The 5-year survival rates for the surgery alone group and the UFT group were 85.4 and $87.9 \%$, respectively, and UFT was found to significantly prolong survival $(p=0.035)$ [4]. In a subsequent meta-analysis of 6 randomized studies comparing UFT as the postoperative adjuvant chemotherapy for NSCLC with surgery alone, including 2003 patients, UFT contributed to the statistically significant prolongation of survival [5]. In addition, the data of the same study population was analyzed by tumor stage (as defined in the seventh edition of the TNM Classification) and the usefulness of UFT was confirmed in stage IA patients with tumors with a diameter (T) of $>2$ to $\leq 3 \mathrm{~cm} \mathrm{[6].} \mathrm{Based} \mathrm{on}$ these results, the Clinical Practice Guideline for Lung Cancer in Japan recommends administration of UFT to patients with completely resected stage IA ( $\mathrm{T}$ of $\geq 2 \mathrm{~cm}$ ) and stage IB NSCLC [7].

Meanwhile, the proposal of the eighth edition of the TNM Classification (TNM8) was reported and it became operative in 2017. The classification of $\mathrm{T}$ is more detailed in the TNM8 than it was in the previous edition [8], and the new system is expected to change the classification of early NSCLC, the target population for adjuvant chemotherapy, and treatment strategy. In this study, the T categories of the JLCRG study population were classified based on TNM8 criteria and the efficacy of UFT were retrospectively investigated in each subgroup.

\section{Patients and Methods}

We performed a subgroup analysis by T category of the TNM8 using data from the JLCRG study. The study protocol was previously reported [4]. This study was approved by the Ethics Review Board of each participating institution and all subjects gave written informed consent.

Tumors were subgrouped on the basis of the $\mathrm{T}$ category (TNM8) as follows: T1a, $\mathrm{T} \leq 1 \mathrm{~cm}$; T1b, $1<\mathrm{T} \leq 2 \mathrm{~cm}$; T1c, $2<\mathrm{T}$ $\leq 3 \mathrm{~cm}$; T2a, $3<\mathrm{T} \leq 4 \mathrm{~cm}$; T2b, $4<\mathrm{T} \leq 5 \mathrm{~cm}$; T3, $5<\mathrm{T} \leq 7 \mathrm{~cm}$. The T4 subgroup ( $\mathrm{T}$ of $>7 \mathrm{~cm}$ ) was excluded from the analysis because no patient in the UFT group had a T4 tumor.

The overall survival was defined as the time from the date of surgery to the date of death from any cause. Alive patients were censored at the date they were last known to be alive. The KaplanMeier method was used for survival estimation. The Cox proportional hazards model was used for the estimation of hazard ratio (HR) and 95\% confidence interval.

\section{Results}

Full details of the JLCRG trial have been previously published [4]. Of the 973 patients, 41 had T1a (UFT, $n=$ 18; control, $n=23$ ), 371 had T1b (UFT, $n=190$; control, $n=181$ ), 344 had T1c (UFT, $n=174$; control, $n=170$ ), 147 had T2a (UFT, $n=74$; control, $n=73$ ), 50 had T2b (UFT, $n=25$; control, $n=25$ ), and 20 had T3 (UFT, $n=$ 10; control, $n=10$ ), respectively. Age, sex, and ECOG performance status did not differ significantly between these 2 groups in each T category.

UFT had an overall survival benefit compared with the control, as shown in Figure 1 and online supplementary Figure 1 (see www.karger.com/doi/10.1159/000479084 
for all online suppl. material). The adjusted HRs were as follows: for T1a, 0.79 (95\% CI 0.14-4.50); for T1b, 1.16 (95\% CI 0.63-2.12); for T1c, 0.74 (95\% CI 0.43-1.27); for T2a, 0.45 (95\% CI 0.21-0.96); for T2b, 0.55 (95\% CI 0.103.07), and for T3, 0.70 (95\% CI 0.20-2.50).

The 5-year survival rates in the control and UFT groups were, respectively, 82.2 vs. $88.2 \%$ for T1a; 92.2 vs. $88.6 \%$ for T1b; 86.3 vs. $88.9 \%$ for T1c; 76.0 vs. $86.2 \%$ for T2a; 84.0 vs. $96.0 \%$ for T2b, and 50.0 vs. $50.0 \%$ for T3.

\section{Discussion}

Taking into consideration the release of the TNM8, we performed an exploratory analysis of data from the JLCRG study and found a tendency toward better outcomes with UFT in all subgroups other than T1b. Data of the subgroups with better outcomes, namely T1c, T2a, and T2b, were analyzed to determine the heterogeneity of the UFT effect on outcome in each subgroup. Exclusions from this analysis were the T1a subgroup because it was not a target of postoperative adjuvant chemotherapy, the T1b subgroup because UFT was not expected to have an effect in this subgroup, and the T3 subgroup because it was small. The heterogeneity test showed that UFT had a similar effect in the T1c, T2a, and T2b subgroups $(p=0.570)$. T1cN0M0, T2aN0M0, and T2bN0M0 correspond to stages IA3, IB, and IIA of the TNM8 and UFT could be considered as a treatment option in these populations. In Japan, the JCOG0707 study is currently investigating the efficacy and safety of UFT and S- 1 (TS- $1^{\circledR}$, a combination preparation of tegafur, gimeracil, and oteracil potassium) as a postoperative adjuvant chemotherapy for early NSCLC [9]. The interim results of a 2-year survival rate and 2-year relapse-free survival rate of the 2 groups combined were reported to be very good ( 97.3 and $89.6 \%$, respectively) [10]. The result of the JCOG0707 study will suggest the need for future assessment of not only UFT, but also S-1 as postoperative adjuvant chemotherapy for early NSCLC.

This study had some limitations, including that the analysis was only exploratory, targets were only adeno- carcinomas, subgroup classification was based only on tumor size and not on invasive size, and the numbers of patients in some subgroups were too small. It was reported that the recurrence rate of adenocarcinoma differs according to pathological classification, and prognosis is better in patients with adenocarcinoma of the lepidic, papillary, and acinar predominant types than in patients with adenocarcinoma of the micropapillary and solid predominant types [11]. In the future, the efficacy of postoperative adjuvant chemotherapy will also need to be investigated in terms of pathological classification because sensitivity to chemotherapy depends on not only the $\mathrm{T}$ category, but also the pathological classification [12]. The JCOG0707 study can investigate the efficacy of S-1 in terms of pathological classification.

In conclusion, an explanatory subgroup analysis of the JLCRG study data demonstrated that UFT tended to improve survival in patients with adenocarcinoma of the lung of each T category based on the TNM8, except for the $\mathrm{T} 1 \mathrm{~b}$ subgroup.

\section{Acknowledgements}

We thank the investigators and all the patients and their families who participated in this study. This work was supported by Taiho Pharmaceutical Co. Ltd.

\section{Disclosure Statement}

M. Tsuboi received honoraria from Taiho Pharmaceutical Co. Ltd.. C. Hamada and H. Kato received consulting fees from Taiho Pharmaceutical Co. Ltd. M. Ohta has no conflicts of interest to declare.

\section{Author Contributions}

All authors drafted and reviewed the report, and approved the final version for submission. Taiho Pharmaceutical Co. Ltd. was involved in data collection, data analysis, and reviewed the manuscript before submission.

\section{References}

Survival Effect of UFT in Lung

Adenocarcinoma
1 Center for Cancer Control and Information Services, National Cancer Center: Cancer Statistics in Japan 2015. http://ganjoho.jp/en/ professional/statistics/brochure/2015_en. html (accessed July 28, 2016).

2 Ferlay J, Soerjomataram I, Dikshit R, et al: Cancer incidence and mortality worldwide: sources, methods and major patterns in GLOBOCAN 2012. Int J Cancer 2015;136:E359-E386.
3 Sawabata N, Miyaoka E, Asamura H, et al: Japanese lung cancer registry study of 11,663 surgical cases in 2004: demographic and prognosis changes over decade. J Thorac Oncol 2011;6:1229-1235.

4 Kato $\mathrm{H}$, Ichinose $\mathrm{Y}$, Ohta $\mathrm{M}$, et al: A randomized trial of adjuvant chemotherapy with uracil-tegafur for adenocarcinoma of the lung. $\mathrm{N}$ Engl J Med 2004;350:1713-1721. 
5 Hamada C, Tanaka F, Ohta M, et al: Metaanalysis of postoperative adjuvant chemotherapy with tegafur-uracil in non-small-cell lung cancer. J Clin Oncol 2005;23:4999-5006.

6 Hamada C, Tsuboi M, Ohta M, et al: Effect of postoperative adjuvant chemotherapy with tegafur-uracil on survival in patients with stage IA non-small cell lung cancer: an exploratory analysis from a meta-analysis of six randomized controlled trials. J Thorac Oncol 2009;4:1511-1516.

7 Japan Lung Cancer Society: Clinical practice guideline for lung cancer. http://www.haigan. gr.jp/modules/guideline/index.php?content id=3 (accessed July 28, 2016).
8 Goldstraw P, Chansky K, Crowley J, et al: The IASLC Lung Cancer Staging Project: Proposals for revision of the TNM stage groupings in the forthcoming (eighth) edition of the TNM Classification for Lung Cancer. J Thorac Oncol 2016;11:39-51.

9 Shirasaka T, Nakano K, Takechi T, et al: Antitumor activity of $1 \mathrm{M}$ tegafur- $0.4 \mathrm{M} 5$-chloro-2,4-dihydroxypyridine-1 M potassium oxonate (S-1) against human colon carcinoma orthotopically implanted into nude rats. Cancer Res 1996;56:2602-2606.

10 Kunitoh H, Tsuboi M, Sakurai H, et al: Safety and compliance data of the phase III study of adjuvant chemotherapy for patients (pts) with completely resected, pathological (p-) stage I ( $\mathrm{T} 1>2 \mathrm{~cm})$ non-small cell lung cancer (NSCLC): a Japan Clinical Oncology Group Trial, JCOG0707 (abstract). J Clin Oncol 2016;34(suppl):8522.
11 Yoshizawa A, Motoi N, Riely GJ, et al: Impact of proposed IASLC/ATS/ERS classification of lung adenocarcinoma: prognostic subgroups and implications for further revision of staging based on analysis of 514 stage I cases. Mod Pathol 2011;24:653-664.

12 Tsao MS, Marguet S, Le Teuff G, et al: Subtype classification of lung adenocarcinoma predicts benefit from adjuvant chemotherapy in patients undergoing complete resection. J Clin Oncol 2015;33:3439-3446. 